\title{
BASIC VARIETY E INTERLINGUA IN ITALIANO L2. NOTE SULLA SCRITTURA DI ARABOFONI
}

\author{
Yabis Martari ${ }^{1}$
}

\section{INTRODUZIONE}

All'interno di un più ampio percorso di ricerca intorno al concetto di basic variety (nell'accezione di Klein e Perdue, 1997, da qui in poi BV) il primo passo che abbiamo fatto, in un lavoro precedente (Martari, 2021), è stato rileggere questo modello nella sua più fruttuosa interpretazione funzionalista e in un orizzonte glottodidattico, contribuendo così a distinguerlo dall'universo generativista con il quale esso ha spesso dialogato, soprattutto nella fase iniziale della sua elaborazione (Meisel, 1997). Il secondo obiettivo (Martari, in preparazione) è stato descrivere il concetto di varietà di base nel quadro epistemologico della teoria della complessità, come "emergenza comunicativa" (intesa propriamente come un'entità complessa con proprietà diverse dalle proprietà delle sue singole parti). Il terzo punto di interesse di questo percorso è applicare il paradigma della BV per leggere contesti linguistici e acquisizionali particolari (per la complessità del quadro sociolinguistico del repertorio linguistico di partenza), come quello dell'italiano $\mathrm{L}^{2}$ di arabofoni. Proprio in questa ultima direzione si colloca il presente contributo. Specifichiamo subito che non vorremmo qui descrivere compiutamente i tratti dell'italiano basico di arabofoni quanto piuttosto proporre una riflessione sul ruolo che la conoscenza di tali tratti può avere in termini glottodidattici. Sottolineiamo inoltre, e con forza, la dimensione di work in progress di questo lavoro che si configura all'interno di una ricerca più ampia, come si è detto, in parte realizzata e in parte ancora da realizzare.

Il presente studio è strutturato come segue. A partire da una premessa il più possibile sintetica su alcune caratteristiche della lingua araba e su poche questioni glottodidattiche essenziali e funzionali al discorso, procederemo con alcune osservazioni sulla BV e ci focalizzeremo infine sulla lettura di corpus testi prodotti in interlingua in italiano L2 di arabofoni che rappresentano un sottocorpus di VALICO (Corino e Marello, 2017).

Le due domande di ricerca alle quali cercheremo dunque di dare una prima risposta, alla fine di questo contributo, sono le seguenti. Gli elementi di interferenza (quelli più classicamente presenti nella letteratura sull'argomento) nell'italiano L2 di arabofoni, come si collocano rispetto alla basicità dell'interlingua? Ostacolano la funzionalità comunicativa - e quindi devono essere assenti nella BV in italiano L2 di arabofoni - oppure non ostacolano la funzionalità comunicativa e quindi sono accettabili in una BV dell'italiano? La seconda domanda, più generale e in certa misura consequenziale alla prima, è invece la seguente: il paradigma della BV è in questo caso influenzato direttamente dal transfer linguistico oppure è più strettamente legato, come vuole il modello originale, alla

\footnotetext{
${ }^{1}$ Alma Mater Studiorum - Università di Bologna.

${ }^{2}$ In linea con le più recenti considerazioni teoriche per la didattica delle lingue straniere, faremo riferimento qui, con L2, a un contesto neutro che «in sé sussume i significati e i possibili utilizzi sia di lingua seconda che di lingua straniera» (Villarini, 2021: 26). Con la precisa indicazione che quasi tutti i testi di VALICO sono stati prodotti in contesto guidato e prevalentemente LS, ovvero da apprendenti di italiano in contesti linguistici e culturali differenti dall'Italia.
} 
potenzialità espressiva delle forme semplificate della L2? Rispetto a questa seconda questione, molto generale e di grandissima portata per gli studi acquisizionali, ovviamente non potremo che fermarci, in questa sede, ad un assai modesto abbozzo di risposta.

\section{PRESUPPOSTI LINGUISTICI E GLOTTODIDATTICI DELL'ACQUISIZIONE DI ITALIANO L2 DI ARABOFONI}

\subsection{Il diasistema della lingua araba}

Il Modern standard arabic è una lingua che i nativi arabofoni apprendono perlopiù durante la scolarizzazione ed è quindi sempre una "lingua seconda" (L2), più che una "lingua madre" (L1); il che provoca una diffusa e nota situazione di diglossia. Rispetto all'arabo classico, l'arabo moderno standard presenta una grammatica leggermente semplificata e soprattutto molte innovazioni perlopiù lessicali che si inseriscono su una comune base fonologica e morfosintattica. Ma nella percezione dei parlanti arabofoni l'arabo classico e lo standard moderno sono entrambe 'al-'arabiyya al-fuṣha $a^{3}$ (فصحى, "pura"), lingua in comune e di prestigio, in base a un concetto non solo linguistico ma, come è stato notato, ideolinguistico (Larcher, 2008; Suleiman, 2003; Anghelescu, 1993; Salem, 20174). Si è in proposito scritto e detto molto sulla «dominance of language in ideological formulations of national identity in the Arab Middle East» (Suleiman, 2003: 224). Oltretutto, si deve ricordare che la lingua araba classica (di cui la lingua coranica è riconosciuta come l'incontestato paradigma) rappresenta una ricostruzione dal momento che «tanto la poesia arcaica quanto il Corano furono messi per iscritto molto tempo dopo la loro apparizione» (Anghelescu, 1993: 11). Una situazione, questa, non sempre realmente compresa e accettata dal parlante non avvertito e profondamente inserito all'interno dello schema ideologico al quale si è fatto pocanzi riferimento. Ogni arabo scolarizzato vive dunque, come si è detto, una situazione di diglossia con almeno due varietà linguistiche: una "pura" e di prestigio e una funzionale e familiare, tradizionalmente non usata nella scrittura, soprattutto istituzionale, estremamente variabile e ricca di prestiti di termini da altre lingue

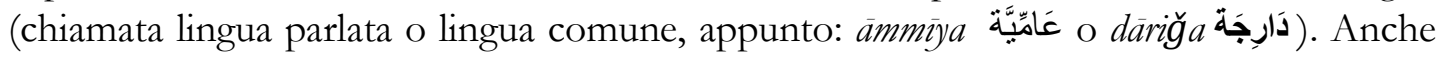
nelle interviste realizzate da Cuzzolin (2004),

è comune a tutti gli intervistati l'idea stereotipa, esplicitamente espressa o lasciata intuire, che la varietà parlata da loro stessi, cioè il loro dialetto, sia una forma corrotta di arabo classico, identificato sempre e soltanto con l'arabo coranico (Cuzzolin, 2004: 101).

Seguendo un modello piuttosto semplificato ma efficace, si può dire che le molte varietà del diasistema della lingua araba possono essere divise in varietà orientali (dall'Iraq all'Egitto) e occidentali (dalla Libia al Marocco), estremamente differenti le une dalle altre ma ritenute, dai parlanti, ugualmente inferiori rispetto alla lingua araba فصحى. Va specificato, tuttavia, che la varietà del Cairo è recentemente percepita spesso come più

\footnotetext{
3 In questo lavoro si ricorrerà a una trascrizione delle parole arabe in caratteri latini il più possibile semplificata; questa scelta, sebbene esponga forse al rischio di qualche imprecisione, consentirà una più facile decifrazione anche al lettore non specialista.

${ }^{4}$ È stato osservato (Salem, 2017) che per le seconde generazioni di arabi in Italia l'arabo tende a diminuire il suo valore con l'avanzamento dell'età: viene cioè considerato sempre meno lingua materna; nello stesso tempo riveste, tuttavia, un valore identitario più significativo nelle fasce di età più avanzate (fra gli universitari e gli studenti della scuola secondaria di II grado ad esempio). Questo aspetto si nota più fra le femmine rispetto ai maschi, e più fra i marocchini, rispetto agli appartenenti ad altri paesi arabi.
} 
prestigiosa (probabilmente in relazione alle diffusissime e ricche produzioni mediatiche). In generale, però, non solo la dignità linguistica ma persino l'identificabilità delle singole varietà è molto spesso negata dagli arabofoni stessi, sebbene le varietà in questione corrispondano spesso a dialetti primari e non secondari rispetto all'arabo del Corano.

Tra parlanti di varietà diverse si usa una varietà mista che tende a semplificare le singole peculiarità fino a raggiungere una forma mediana e intercomprensibile (una sorta di lingua franca); tuttavia non è raro che gli arabofoni migranti impieghino come lingua franca, invece, una lingua occidentale: soprattutto l'italiano e il francese. Da questo quadro, qui rapidamente abbozzato, possiamo già trarre una prima ma importante considerazione: il parlante arabo ha spesso una forte propensione verso una dimensione funzionale della lingua di comunicazione separata tendenzialmente dalla lingua identitaria (arabo standard), percepita per altro come la sola da difendere perché garante dell'unità e quindi della forza del mondo arabo. È immaginabile che per il parlante arabofono, quindi, la potenzialità funzionale di una varietà basica sia facilmente accettabile. In altre parole, almeno per ciò che riguarda la comunicazione orale, informale e quotidiana, forme semplificate, e di conguaglio, sono considerate come pienamente adeguate e non deprecabili, all'interno di una logica di una lingua "di consumo", che non occorre proteggere, né perfezionare, al contrario di quanto accade per lo standard.

\subsection{L'apprendente arabofono nella prospettiva glottodidattica}

Sulla base del contesto indicato in 2.1, dovrebbe già essere chiaro che quando si pensa al parlante arabofono si fa riferimento - soprattutto nel contesto migratorio - a una pluralità di figure. E ovviamente tale variabilità ha un riflesso importante sul piano glottodidattico.

Come ben sintetizzato da Francesca Della Puppa (2006: 56 e sgg.), infatti, a diversi profili linguistici dell'apprendente arabofono corrispondono diversi scenari educativi per l'insegnamento di L2. Tra le tante sfumature possibili possono essere presi a riferimento i seguenti tre casi:

1. arabofono non alfabetizzato che utilizza e conosce solo una varietà non فصى dell'arabo;

2. arabofono alfabetizzato che utilizza una varietà non standard dell'arabo ma anche l'arabo classico e/o standard a livello base;

3. arabofono alfabetizzato che utilizza una varietà non standard dell'arabo, conosce e usa l'arabo classico e/o standard a livello avanzato e sa usare anche una lingua occidentale.

Vale tuttavia anche la classificazione operativa di Bosco (2004: 143), ugualmente basata sul livello di scolarizzazione, ed estesa su 4 gradi.

A questi profili corrispondono dunque certamente esigenze differenti legate anche a diversi gradi e orientamenti della motivazione personale dell'apprendente. Così, un apprendente poco scolarizzato ha molto spesso necessità più funzionali (di primo inserimento nella società), mentre un apprendente molto scolarizzato ha una motivazione anche legata all'approfondimento linguistico e culturale. Pur sulla base di questa ovvia considerazione - sempre da Bosco (2004: 149) - si ricava il dato interessante che la motivazione all'apprendimento non solo della lingua parlata ma anche di quella scritta è forte e diffusa in tutte le tipologie di apprendenti. E, ancora sulla scrittura, Bosco (2004: 150) rileva una diffusa «convinzione del maggior valore di una lingua quando è scritta». Ciò si pone ovviamente in relazione alla ben nota concezione del mondo arabofono 
secondo la quale la scrittura è percepita perlopiù all'interno di un universo di prestigio mentre la lingua orale è legata a contesti funzionali di comunicazione quotidiana. Si tratta di una questione importante e non poco problematica, quella diamesica, che oggi in contesto educativo dovrebbe certamente essere rivista: dal momento che gli apprendenti arabofoni legano certamente alla scrittura un concetto di maggiore prestigio, ma la nuova comunicazione scritta (digitale, quotidiana, non sorvegliata) pone anche questi parlanti nella posizione di ripensare al testo scritto all'interno di nuovi parametri. Riformuleremo meglio questa ipotesi nel paragrafo conclusivo, alla luce dell'analisi dei dati.

Tornando ora, invece, alla figura dell'apprendente arabofono, possiamo certamente sottolineare che un elemento di grande peso da ogni punto di vista glottodidattico è il livello di scolarizzazione. Coloro che sono debolmente, discretamente, fortemente alfabetizzati hanno richieste e bisogni formativi differenti (si veda la sezione di riflessione glottodidattica di Vedovelli et al., 2004), cui devono corrispondere spesso anche tecniche e approcci differenti, sebbene sulla scorta di denominatori culturali e motivazionali comuni e profondi - proprio come, si è detto, è la percezione della scrittura e del testo scritto. Rispetto a questo piano, quello della motivazione, occorre poi tenere conto di un numero importante di variabili e situazioni particolari. Ad esempio, rispetto all'apprendimento della lingua italiana devono essere segnalati, soprattutto in prospettiva LS, casi in cui lo studente arabofono ha, di partenza, una percezione linguistica negativa della lingua target, come ad esempio in Marocco (Eddahani, Dlimi, 2019), fatto che influisce in modo negativo, appunto, sulla motivazione all'apprendimento. $\mathrm{O}$, ancora, casi linguisticamente molto complessi come quello del parlante arabofono e italofono maltese, su cui i lavori di Giuseppe Brincat restano il riferimento principale (da ultimo Brincat, 2021).

Per un quadro completo della dimensione glottodidattica dell'apprendente arabofono di italiano L2 si rimanda senz'altro a Della Puppa (2007: 28), che riporta coordinate metodologiche generali al contesto particolare qui delineato, sintetizzando opportunamente quanto segue:

Il codice dovrebbe distinguere gli errori che l'alunno avrebbe potuto evitare rispetto a quelli inevitabili perché pre-sistematici, cioè errori dovuti alla non conoscenza della regola.

Inoltre, dal momento che una parola spesso contiene errori di diverse tipologie è necessario utilizzare un codice che possa in modo chiaro e immediato far cogliere all'allievo la diversità tra errori di ortografia, di morfologia, di sintassi e di lessico.

Una volta evidenziati gli errori, si può procedere chiedendo all'allievo di cercare di autocorreggersi, senza fornire subito il feedback, corretto. Se l'allievo è in grado di farlo, sappiamo che si è trattato di errori post-sistematici, ovvero di devianze dovute alla distrazione o alla fretta nella scrittura, ad una mancata funzione del monitor piuttosto che ad una non comprensione o non conoscenza della regola.

\subsection{Alcune caratteristiche della lingua araba che producono interferenza nell'interlingua di apprendenti di italiano L2}

Non descriviamo qui che pochissime caratteristiche della lingua araba standard, soffermandoci sugli elementi di interferenza interlinguistica classicamente osservati in apprendenti di italiano L2 (per la descrizione delle caratteristiche dell'arabo standard, talora in relazione alle varietà parlate, salvo diverse indicazioni il rimando è alle sistematizzazioni di Ryding, 2005; Brustad, 2000 e Holes, 2004). Anticipiamo inoltre che 
tali elementi devono essere considerati, almeno nel contesto glottodidattico, allinterno di un quadro più ampio: come emerge anche dalla lettura del nostro corpus, ci sono infatti meccanismi di interferenza e di prestito da altre lingue - soprattutto parole cosiddette "tappabuchi" (Corino, Marello, 2017: 105) dallo spagnolo, francese e inglese, come si vede ad esempio in $\mathrm{t} 25, \mathrm{t} 38$ e t56 in appendice - che si innescano abbastanza frequentemente e che complicano il quadro non solo acquisizionale ma anche linguistico-educativo dell'apprendente arabofono.

Da ultimo, specifichiamo che le note di questo paragrafo - lungi dal rappresentare un qualsivoglia contributo all'arabistica - costituiscono soltanto un'approssimazione estrema di alcuni fenomeni significativi del Modern standard arabic. Occorre oltretutto essere ben consapevoli che tale varietà si colloca come una parte non ampia, in fondo, del grande diasistema della lingua araba; tuttavia - essendosi lo standard posto, per lungo tempo, come denominatore comune della lingua scritta degli arabofoni - crediamo che questa limitatissima disamina sia utile al lettore come indispensabile orientamento all'interno del nostro discorso che, lo ricordiamo, è finalizzato a focalizzare la relazione tra interlingua e BV. Si assume poi di conseguenza, come implicito, che le caratteristiche dell'interlingua, in un contesto così variegato e complesso come quello qui indagato, siano certamente influenzate non soltanto dell'interferenza del codice standard, ma anche e forse soprattutto delle varietà parlate dell'arabo (cioè, a conti fatti, le vere e uniche lingue madri degli arabofoni: cfr. Abi Aad, 2006), così come, lo ribadiamo, di altre lingue precedentemente apprese. Queste osservazioni dovrebbero suggerire che ogniqualvolta parleremo, nel presente articolo, di "ipotesi di interferenza", il riferimento non dovrà essere unicamente al passaggio dalla lingua madre all'italiano come L2, quanto piuttosto dall'intero repertorio dello scrivente (composto dalla varietà/lingua madre, dallo standard e dalle eventuali altre lingue) all'italiano come lingua successivamente appresa (che può rappresentare dunque la $\mathrm{L} 3$, la $\mathrm{L} 4$, etc.); ovviamente cercando di considerare nel modo più esclusivo possibile i fenomeni (di seguito elencati e commentati) tipicamente attribuibili a un parlante e scrivente arabo.

Partendo com'è d'obbligo dal livello fonologico e fonetico (per il quale resta fondamentale il rimando a Bernini, 1988), occorre subito ricordare che il sistema consonantico dell'arabo standard presenta suoni inesistenti in italiano e nelle lingue europee (come i foni gutturali $\varepsilon, \dot{\varepsilon}$, ق rispettivamente uvulo-velare, post-velare, faringale), ma anche la neutralizzazione di coppie minime ( $p$ e $v>b$ e $f$ ) e lacune (zaino, ciao, cagnolino, lughio), oltre a variazioni grafiche importanti: la distinzione grafica di $s$ sorda e sonora ( $\mathrm{w}$ e $j$ ), non esistono geminate grafiche e quindi, di fatto, le doppie esistono solo come suono. Il sistema vocalico è ufficialmente limitato ai tre suoni $a, u, i$ (brevi e lunghi, e quando brevi graficamente inseriti come diacritici non propriamente alfabetici): di fatto, dunque, nel sistema fonetico del parlante arabo il suono $a$ è considerato quasi omofono di $e$ il suono $u$ è considerato quasi omofono di $o$. Quindi $e$ e $o$ non costituiscono due fonemi ${ }^{5}$. Esistono, poi, nel Modern standard arabic, solo due dittonghi (au, ai). Ovviamente questa e le seguenti affermazioni devono essere considerate come forti generalizzazioni e circoscritte, lo si ribadisce, allo standard: diversa è cioè la situazione nelle molte varietà, anche (non solo) dal punto di vista fonetico.

Questo primo elenco di fenomeni, prevedibilmente, produce interferenza (se non altro in senso ampio, cioè la difficoltà di produzione di una forma in L2 perché assente in L1). Si aggiunga poi che in arabo le parole non iniziano mai con due consonanti: ciò fa sì che

\footnotetext{
${ }^{5}$ È poi anche più rilevante specularmente, ovvero per l'arabo appreso da italiani, il fatto che quasi tutte le lettere possono avere quattro realizzazioni grafiche (iniziale, in corpo di parola, finale, isolata). Lo stesso dicasi per altri fenomeni, come il fatto che esistono in arabo maschile e femminile non solo della $3^{\text {a }}$ ma anche della $2^{a}$ persona (singolare e plurale).
} 
gli arabofoni tendano a inserire in italiano L2 un suono vocalico prima o tra le due consonanti inziali.

Un ulteriore elemento che interferisce negativamente nella produzione e nella ricezione in italiano L2 di un arabofono è l'aspetto grafico del testo: l'esistenza cioè, nella scrittura, del solo corsivo, e l'assenza di maiuscole. Ed è zona altresì problematica quella della pronuncia dei numeri, dal momento che, per il parlante arabofono, le decine devono essere sempre lette prima delle unità ${ }^{6}$.

Morfologicamente l'arabo è una lingua introflessiva, con tre categorie principali (nome, verbo e particella), ed è caratterizzata da un largo uso di infissazione. Le parole sono quindi costruite dall'incastro di una delle circa 6000 radici consonantiche (quasi sempre triconsonantiche) con le vocali (es. CvCvC: e così, dalla radice كتب KTB > Katb (scrittura), Kàtib (scrittore) etc). Le parole sono lemmatizzate con la radice (che per il verbo corrisponde alla terza persona del perfetto).

Il sistema della declinazione, che individua caso diretto, indiretto e obliquo (con un cambio di vocale alla fine del nome) non è quasi in uso nelle varietà ma persiste nello standard.

Dei due generi, è marcato il femminile ma non il maschile (dottore/dottoressa: طبيب tabib, tabiba) e ci sono tre numeri, compreso il duale; anche in questo caso, in realtà, quest'ultimo è poco usato nel sistema verbale delle varietà ed è perlopiù riservato agli elementi sempre in coppia come mani, piedi, occhi etc.

L'articolo determinativo ha una forma unica $(a b)$ e non esistono indeterminativi, il che crea problemi di acquisizione e distinzione di paradigmi più ricchi, come quello della lingua italiana. Inoltre, anche la separazione dell'articolo dal nome (non presente nella forma araba) è un elemento di ricorrente difficoltà nell'interlingua di arabofoni.

Un ulteriore elemento classicamente individuato come motivo di interferenza nell'apprendimento di altre lingue è la presenza dell'articolo dopo l'aggettivo dimostrativo: ad esempio: hadhih alfata (الفتاة هذه), lett. 'questa la ragazza'.

È poi rilevante in ottica acquisizionale anche il fatto che in arabo la funzione del possesso sia espressa perlopiù da pronomi personali e suffissi che si accordano con il possessore.

Restando sul piano morfologico, un elemento di grande peso nell'apprendimento di altre lingue è che in arabo i verbi hanno funzione prevalentemente aspettuale e non deittica. È stata in proposito studiata la difficoltà crescente nell'uso di presente $<$ futuro $<$ passato, nel controllo del verbo in italiano (cfr. Al-Ali, Al-Oliemat, 2008). Ma soprattutto si segnala per l'italiano L2 l'uso spesso sovraesteso dell'imperfetto, proprio come risultato del netto primato della funzione aspettuale su quella deittica e della scarsa frequenza di forme perfettive in contesto subordinativo nella L1.

Dal punto di vista sintattico, l'arabo è una lingua classicamente VSO (es. 'il ragazzo ha incontrato un amico' > altaquaa (ha incontrato) alsabiu (il ragazzo) bisadiq (un amico) (التقى) (.). Tuttavia, nello standard moderno e nelle varietà contemporanee tende ad avvicinarsi più frequentemente a strutture SVO, soprattutto per influenza del modello delle lingue occidentali come italiano, francese, inglese.

Significativo dal punto di vista tipologico e potenzialmente interessante da quello glottodidattico è che l'ordine prevalente sia Determinato-Determinante: "La bella ragazza" > alfatat (la ragazza) aljamila (la bella) الفتاة الجميلة

Se, proprio come in italiano, l'arabo consente costruzioni con soggetto 0 perché il verbo presenta morfemi che consentono di risalire alla persona, è invece un elemento di distanza il fatto che non esista il verbo "essere" al presente con funzione copulativa (mentre ovviamente esistono forme corrispondenti per il passato e per il futuro). E può

${ }^{6}$ Talora i parlanti arabofoni usano i numeri indiani - 1 r r etc. 
rappresentare un elemento significativo dal punto di vista acquisizionale (Bettega, Russo, 2017) il ricorso al "participio attivo" il quale si presenta come la seconda alternativa (oltre al costrutto nominale senza copula) al verbo in forma esplicita: una forma anch'essa nominale e senza alcuna marca temporale né aspettuale.

Rispetto al sistema verbale è stata anche valutato il peso della tense neutralization (Bettega, Russo, 2017: 43 e sgg.): in base a questo costrutto, il tempo passato viene espresso lessicalmente solo a inizio enunciazione e poi - nelle eventuali forme successive - si usa il presente. Si costituisce di conseguenza come elemento di interferenza da parte del sistema verbale della L1 in italiano la sovraestensione del presente (sia come frutto di neutralizzazione vera e propria, sia come traduzione di forme in cui in L1 non è previsto un costrutto verbale esplicito).

Altro elemento di potenziale perturbazione - ancora dal punto di vista sintattico - è il seguente: per la frase relativa, in arabo si differenziano le costruzioni di relative con antecedente determinato o indeterminato (in questo secondo caso il relativo è inespresso e per la grammatica araba il costrutto non è realmente ascrivibile a quello della relativa) e in base al caso (soggetto, diretto, indiretto). Nel caso in cui sia oggetto ha anche ripresa ("ritornante"), nel caso in cui sia indiretto si presenta come antecedente invariabile (come la costruzione italiana con relativo indeclinato "l'uomo che hai parlato con lui è mio padre"). Il segnalatore doppio del complemento diretto compare anche come transfer nell'interlingua.

Ancora qualche parola per riprendere meglio, alla fine di questa stringatissima rassegna, l'espressione del possesso in arabo. Il verbo "avere" non esiste quasi mai e si utilizzano perifrasi di luogo, compagnia, attribuzione. Per il possessivo spesso di usa lo "stato costrutto" (complemento di specificazione per giustapposizione. Ad esempio, "Bin Laden" > figlio [di] Laden).

In sintesi, dunque, sono state rilevate - soprattutto ma non solo in approcci contrastivi - zone di difficoltà di apprendimento della lingua italiana con possibilità di interferenza nelle seguenti aree:

- Sistema vocalico e dittongazione

- Perturbazione e resa grafica consonantica (es. $v>b, g l>l$ )

- Geminate

- Posizione/uso articolo

- Coniugazione e uso del sistema verbale complesso dell'italiano

- Sovraestensione participio ("participio attivo")

- Sovraestensione imperfetto

- Funzione deittica del verbo (difficoltà presente $>$ futuro $>$ passato)

- Ordine Determinato-Determinato

- Uso copula del verbo

- Tense neutralization

- Uso verbo avere

- Possessivo e "stato costrutto"

- Frase relativa

Questa lista recepisce le indicazioni dei principali studi, più o meno noti, le seleziona e le integra, dal momento che le caratteristiche dell'interlingua italiana di nativi arabofoni individuate nei diversi lavori sull'argomento in esame non coincidono affatto. Talora perché focalizzati su di un solo fenomeno, talaltra perché troppo genericamente orientati 
alle cosiddette "difficoltà di arabofoni" e quindi comprensive di questioni più generali, non chiaramente legate al sistema linguistico della $\mathrm{L}^{7}$.

Tuttavia, tale elenco non ha affatto la pretesa di essere esaustivo e non esclude che ci siano interferenze caratteristiche (legate sia allo standard, sia alle varietà ${ }^{8}$, sia al singolo parlante) che qui non abbiamo preso in considerazione. Inoltre, ci sono piani linguistici più sofisticati (come quello retorico o quello pragmatico: ad esempio in arabo non si usa il "lei" e solo raramente il "voi") che, in considerazione del tipo di osservazione che qui proponiamo restano necessariamente solo sullo sfondo. Resta dunque il riferimento inevitabile al lavoro di Della Puppa (2006, 2007, 2008, 2009) per una disamina più approfondita e soprattutto estesa di ciò che concerne l'influenza della L1 sull'interlingua di arabofoni apprendenti italiano come L2. Sono poi utili anche le considerazioni di Boussetta (2006) sul lessico in prospettiva culturale («analizzando i testi scritti da certi studenti arabofoni si capisce quanto sia difficile fare ricorso alla traduzione letterale perché le parole o le espressioni arabe tradotte letteralmente in italiano cambiano totalmente il significato del testo e rendono poco comprensibile il messaggio»), e sulla testualità come organizzazione retorica culturalmente determinata in relazione a particolari strutture, selezioni, organizzazioni delle informazioni. In questi testi, sebbene non sia oggetto del nostro studio, è talora possibile osservare un insieme di questioni interculturali che individuano - per dirla con Balboni (1999) - "parole comuni e culture diverse".

\section{Il CONCETTO DI BASICO/PREBASICO/POSTBASICO}

Questa sezione, ancora più sintetica della precedente, non rappresenta in alcun modo una trattazione teorica della categoria di BV, né un excursus sul dibattito intorno a questo discusso modello, ma solo la fissazione di alcuni punti indispensabili al nostro discorso.

Quello di BV è un concetto funzionale, quindi relativo a ciò che un enunciato può fare, non a come è formato. Tuttavia, sulla scorta di un una tradizione consolidata di studi (si veda ad esempio Giacalone Ramat, 2003 e Bernini, 2005), è nostra convinzione che esso possa essere però riportato a fenomeni linguistici di semplificazione, conguaglio e riduzione relativi a diverse lingue, ma in certa misura comuni (Martari, 2021). In ottica funzionalista, è una varietà di base quella che permette con il minimo di complicazione formale la massima resa in termini di potenzialità espressiva, anche di concetti complessi.

Con le parole di Klein e Perdue (1997: 304) possiamo infatti definire BV

a relatively stable system to express themselves which

- seemed to be determined by the interaction of a small number of organisational principles

- was largely (though not totally) independent of the specifics of source and target language organisation

- was simple, versatile, and highly efficient for most communicative purposes.

\footnotetext{
7 Ad esempio, in Elgheriwi (2017) è indicata come zona di interferenza una generica "difficoltà a formulare frasi complesse"; tale questione è tuttavia riportabile non tanto a un generale fenomeno di interferenza (è al limite il risultato della presenza di molti e specifici fenomeni di interferenza) quanto piuttosto alla difficoltà di padroneggiare strutture complesse in una L2.

${ }^{8}$ Sarebbe interessante, ad esempio, riflettere sul fatto che il cosiddetto Agreement Neutralization (Brustad, 2000: 62 e sgg.) abbia influenza su alcune costruzioni nella L2, ma non essendoci indicazioni in letteratura acquisizionale italiana su questo, nel presente lavoro non possiamo prendere in considerazione tale fenomeno. «Neutralization of adjectival agreement is prevalent in Egypt among certain classes of adjectives, and a few isolated cases may be found in the Levant and Kuwait» (Brustad, 2000: 62).
} 
Di conseguenza, sarà da considerarsi prebasico ciò che non è ancora funzionale (e che quindi non consente al parlante di esprimere ciò che desidera) e postbasico ciò che, oltre a produrre la comunicazione desiderata, impiega strumenti più raffinati di espressione $\mathrm{e}$ "strategie di trasparenza" (Giacalone Ramat, 2003: 349): per l'italiano, ad esempio, la morfologia verbale e la specializzazione delle strutture subordinative.

La categorizzazione della BV non è rigida: ci sono fenomeni che permangono in continuità tra l'uno e l'altro livello della competenza. E ciò accade soprattutto perché le strategie prebasiche riemergono come risorsa comunicativa quando la strumentazione basica non è adeguata, perlopiù come tentativi di colmare (con strumenti inefficaci) lacune del sistema basico, talora attingendo al repertorio della L1 o di altre lingue.

Come si è cercato di suggerire altrove (Martari, 2021), sebbene il paradigma della BV si riferisca a un universo acquisizionale spontaneo, nel momento in cui esso viene impiegato come criterio esplicito di funzionalità (in linea, ad esempio, con la nota schematizzazione di Bernini, 2005: 125), tale paradigma potrebbe essere impiegato come strumento descrittivo anche per un contesto guidato - proprio come accade utilmente con la prospettiva dell'interlingua per l'insegnamento linguistico proposta da Pallotti (2017). Ci pare tuttavia che, soprattutto per il contesto dell'insegnamento, sarebbe più giusto rappresentare in modo esplicitamente relativo la competenza linguistica in base al criterio della BV. Ad esempio, attraverso un diagramma (Figura 1) che rappresenti forme basiche, e pre- e postbasiche come compresenti in tutte le VdA ma in quantità variabili in base al livello di competenza linguistica, così da produrre classificazioni di testi tenden₹ialmente basici, prebasici o postbasici:

Figura 1. Ipotesi generale di distriburione delle forme basiche e pre- e post-basiche all'interno di testi giudicati basici e pre e post-basici

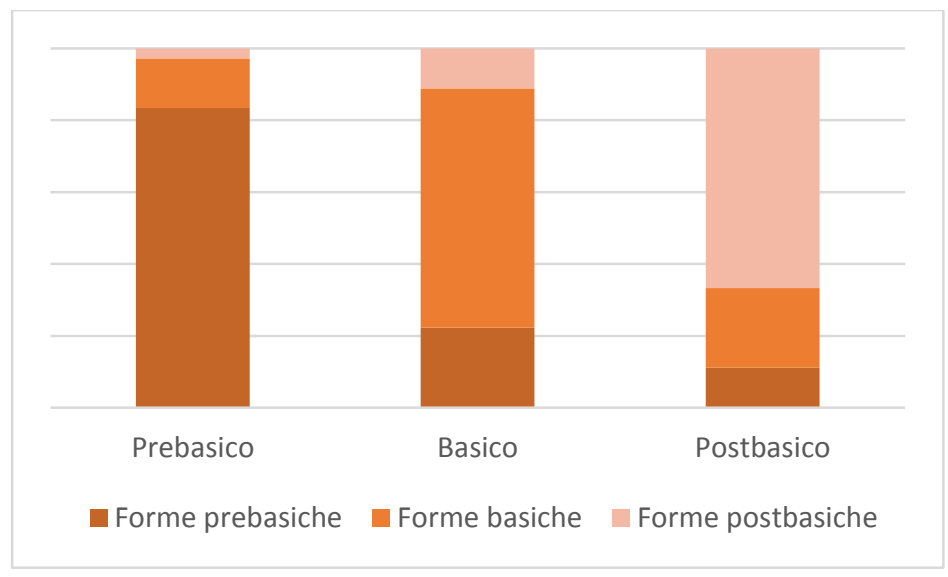

Se ciò è teoricamente valido per il contesto spontaneo, tanto più lo sarà per la dimensione dell'apprendimento guidato, e dunque inevitabilmente supportato da spiegazioni grammaticali e lungo un curriculum che certamente non può ignorare la morfologia. Com'è stato osservato da Elisa Corino - proprio in relazione all'analisi dei testi raccolti nel corpus VALICO - infatti, «i risultati non sono sempre omogenei e sovrapponibili, anzi, talvolta le osservazioni a partire da VALICO (e corpora siffatti, come ADIL2), divergono parzialmente dalla grammatica dell'italiano L2 descritta dalla ricerca acquisizionale» (Corino, Marello, 2017: 11).

Qualche esempio di questa sfocatezza e compresenza di forme all'interno di testi tendenzialmente (pre/post)basici. Anche in alcuni testi del nostro sottocorpus (ad esempio il testo [t23] in appendice), si vede bene come ci siano molti elementi postbasici ma come 
la parte finale del testo sia incomprensibile e contenga elementi prebasici (cioè, in questo caso, elementi lessicali inesistenti). Oppure, ancora, se leggiamo un enunciato come "gli due cane sfruttare lacazzione è mangiare tutti li cose" [t30], gli elementi di mancato controllo morfologico rapportabili alla dimensione della BV (accordo sbagliato e verbo non coniugato) non sono quelli che rendono il testo incomprensibile.

Ancora qualche considerazione, per così dire, en passant, indispensabile a introdurre la nostra prospettiva. Innanzitutto, è bene specificare che anche il concetto di funzionalità della BV è meno univoco di come può sembrare. La funzionalità di una comunicazione si basa infatti su di una collaborazione tra gli attori nel processo comunicativo: possiamo definire un testo efficace solo in relazione al fatto che l'interlocutore lo ritenga accettabile, cioè che accetti di impiegare le regole del sistema interlinguistico del parlante/scrivente. $\mathrm{E}$, in proposito, si deve notare che, se fosse adottata esclusivamente la prospettiva del madrelingua (pressoché di qualsiasi madrelingua), quello che è basico non rispecchierebbe mai alcun criterio di accettabilità; invece accade che il madrelingua accetti le regole dell'enunciato del parlante non nativo che utilizza forme di BV. Del resto, focalizzando ancora di più il problema, anche il criterio di accettabilità deve essere precisato: ci sono infatti motivi diversi per cui un lettore può ritenere non accettabile un testo. Nelle varietà di base si tratta spesso di ragioni legate alla riduzione del sistema morfosintattico, quindi elementi che di fatto diminuiscono (senza però impedirla) la comprensibilità del testo. Questi motivi, nella comunicazione quotidiana, salvo casi rari, sono perlopiù assenti, così la BV è "sufficiente" per svolgere molte delle funzioni della comunicazione verbale. Ovviamente, dunque, criteri come funzionalità o accettabilità dipendono non solo dalle competenze e dalle caratteristiche del lettore e dello scrivente ma, come per qualsiasi altro evento comunicativo, dipendono anche dalle condizioni diafasiche e dal contesto (in un momento e date certe condizioni, dunque, un parlante può ritenere accettabile "impegnarsi" nella lettura e nella produzione di un testo foreigner talk, mentre in altre no). E dipende anche da condizioni diamesiche, perché la comprensibilità di un testo scritto $\mathrm{O}$ orale (anche se letto) è diversa, e così l'impegno richiesto al ricevente (e la possibilità che sia accettato e compreso) è variabile. Tutto ciò, appunto, rende estremamente sfocata anche la categoria stessa di BV.

Queste precisazioni, lo si ribadisce, non indicano che non ci sono elementi caratteristici di una varietà di base (così come di una varietà pre- o postbasica). Significano piuttosto che occorre ipotizzare sempre una certa sfocatezza nella categorizzazione di cosa può essere considerato BV.

Infine, vale la pena di ricordare che, proprio rispetto agli errori di morfologia, Della Puppa (2007: 24) ha notato, su di un corpus diverso, quanto «questo tipo di errori talvolta compromette la comprensione dell'enunciato». In questa prospettiva, dunque, una varietà di base può forse anche essere definita come il punto in cui l'accettabilità è tale da essere funzionale e quindi consentire il processo comunicativo. In chiave glottodidattica

il grado di inaccettabilità del testo, però, non deve ritorcersi contro l'alunno, ma deve piuttosto stimolare il docente a cogliere gli errori più significativi sui quali lavorare con lo studente perché possa prima possibile essere messo nelle condizioni di scrivere un testo con un'efficacia comunicativa maggiore (Della Puppa, 2007: 29).

\section{ANALISI DI SCRITTII IN ITALIANO DI ARABOFONI}

Estraiamo e analizziamo, ora, un sottocorpus di scritti di arabofoni a partire dal corpus di apprendenti di italiano VALICO. Si tratta di 60 testi; non molti, però significativi per 
distribuzione e tratti dal learner corpus più autorevole, meglio bilanciato e meglio strutturato per la lingua italiana. Per i dettagli ampiamente discussi della formazione del corpus si rimanda a Corino e Marello (2017), e per alcuni studi significativi a partire da VALICO si rimanda invece al volume curato da Corino e Onesti (2017) ${ }^{9}$.

\subsection{Il corpus e i dati}

Abbiamo classificato la funzionalità basica degli enunciati in relazione a un giudizio di comprensibilità da parte di cinque insegnanti di italiano L2, lettori esperti ${ }^{10}$ che hanno valutato i testi, in diretta corrispondenza con le categorie di prebasico, basico, postbasico, in base alla seguente scala:

C. Sono presenti forme lessicali e sintattiche lontane dalla varietà target (VT), la morfologia è perlopiù incongruente con la VT, e il testo, dal punto di vista funzionale, non è comprensibile e quindi inefficace [prebasico]

B. Sono presenti forme lessicali e sintattiche lontane dalla VT ma il testo, dal punto di vista funzionale, è comprensibile, anche con elementi morfologici scarsi e generalmente non congruenti con la VT [basico]

A. Sono presenti forme lessicali e sintattiche perlopiù identificabili con la VT e il testo è comprensibile, con elementi morfologici congruenti con la VT [postbasico]

I problemi di omogeneità del giudizio tra i cinque lettori, sono stati rilevati soprattutto rispetto alla valutazione dell'accuratezza grammaticale (ovvero nella scelta tra classificare un testo come $\mathrm{B}$, basico o A postbasico), ma il giudizio che ci interessa di più, in questo studio, è ovviamente quello di comprensibilità (la scelta tra $\mathrm{C}$ prebasico e $\mathrm{B}$ basico), sul quale i nostri lettori esperti hanno espresso pareri tendenzialmente piuttosto concordanti (Figura 3).

In seconda battuta, nell'annotare il nostro piccolo corpus, abbiamo segnalato alcuni tratti che possono essere considerati ipotesi di interferenza della L1, a partire dalla letteratura prima sintetizzata sull'argomento. Sebbene non si possa considerare questa analisi esaustiva, crediamo sia certamente significativa proprio a livello di formazione di ipotesi, dal momento che i tratti presi in considerazione, come si è visto, non sono pochi. Ci siamo sforzati di escludere, inevitabilmente con un giudizio soggettivo, $i$ casi in cui l'interferenza ci è sembrata soltanto apparente come ("le loro cose e affare" [t31], dove la $i>e$ sembra più una forma analogica per la morfologia del plurale femminile in italiano che un errore legato all'interferenza del sistema vocalico dell'arabo), distinguendoli da probabili vere e proprie interferenze, come ad esempio le seguenti forme: "affaciata alla finestra davante la scena" [t28]. In generale abbiamo poi cercato di scartare $i$ tratti che in alcuni casi vengono presi in considerazione come frequenti in scritti di arabofoni ma che sono del tutto peculiari della complessità del sistema dell'italiano più che della sua lontananza da quello della L1 araba (come, ad esempio, il corretto uso del paradigma preposizionale per le reggenze verbali) e che quindi devono essere riportati principalmente a una dimensione evolutiva.

\footnotetext{
${ }^{9}$ Le molte interferenze presenti nei testi di questo sottocorpus rispetto all'uso dei verbi sono state rilevate e analizzate da Bettega e Russo (2017), cui, di nuovo, si rimanda senz'altro.

${ }^{10}$ I lettori che hanno dato il giudizio di comprensibilità dei testi sono cinque insegnanti di italiano L2 delle scuole della provincia di Bolzano e Modena. La valutazione finale, laddove discordante per i cinque valutatori, è stata assegnata a maggioranza (ad esempio BBBCC $>\mathbf{B}$, cfr. Figura 3).
} 
Ponendo in relazione i giudizi (A, B, C) e le analisi si ottengono testi valutati come i seguenti (Figura 2, in cui vengono indicati in neretto le forme che abbiamo valutato come possibili interferenze):

Figura 2. Esempi di testi estratti dal corpus V ALICO e annotati

\section{A - POSTBASICO (5/5)}

L' altro giorno due uomini camminavano sul marciapiede erano tutti i due carichi di spese che avevano comprato un giovane ragazzo con la sua bicicletta carica di spese che aveva comprato al supermercato ( latte, olio, frutta e anche una salsicia ). Era in compagnia con suo piccolo cane. Rientrava tranquillamente a casa cuando all' improviso ha sbattuto contro un' altro uomo che veniva dalla parte opposta del marciapiede. Era anche lui stracarico di roba diversa sulla sulle spalle, nelli mani, con un cane (anche lui ). Una signora affaciata alla finestra davante la scena di sbattimento dei due uomini ne aprofitta per racogliere $i$ due ( palloni ) palloni . (mentre i due uomini ( non ). Invece di racogliere la loro spesa spersa ? da per tutto, loro si misero a litigare con le salsice.

\section{B - BASICO (4/5)}

Ieri al parco c' era un uomo che leggeva un giornale e che sta guardando una donna che chiede aiuto perché il suo marito l' ha colpisce allora lui è stato arrabiato e ha si ha alzato dal suo banchino e ha colpito il marito ma lui ha Credo che cosi la donna diventa felice ma ha scoperto che era sbagliato perché questa donna era molto arrabiato per il suo marito e si mette a gridare. Perché lei ama molto suo marito e non buole che la il suo aiuto . e che non ha capisce perché

\section{C - PREBASICO $(5 / 5)$}

L' altro giorno due uomini camminvano sul marciapiede . una persona che vendi un lita sul biciclelta porta una cali é un persona chi vado al mari per giochi un cali é due persona chi una problema é bonu che guardavano il spettacole con gli due persona é due cali é tutto gli cose sono cadouti sul gli strada

Abbiamo dunque contato il numero di forme ipoteticamente riportabili a interferenza, anche se l'aspetto quantitativo è di per sé poco preciso perché non è sempre facile discernere quando annoverare un fenomeno tra $i$ transfer oppure no: occorre infatti com'è certamente noto a chiunque si occupi di analisi di interlingua - spesso un intervento interpretativo inevitabilmente soggettivo; inoltre, a volte $\mathrm{i}$ fenomeni si ripetono a maggiore o minore distanza oppure si accumulano nello stesso item lessicale (es. "vendettore" [t30]) ed è necessario scegliere se considerare una sola occorrenza oppure no. Abbiamo infine posto questo dato in relazione con il numero di parole del singolo testo per dare almeno un suggerimento del rapporto tra numero di ipotesi di transfer e quantità di testo prodotto. Per ogni testo è anche indicato il livello di attribuzione finale (A, B , C) e i relativi cinque giudizi dei lettori. Ne è risultata la tabella che segue (Figura 3), in cui le valutazioni dei testi sono riportate in ordine da A (14 testi), a B (26 testi) a C (20 testi): 
Figura 3. Giudizi di comprensibilità dei lettori sui testi analižati, e numero di forme riportabili a interferenza per ogni testo, in relazione al numero complessivo di parole

\begin{tabular}{|c|c|c|}
\hline $14 \mathrm{~A}$ & $26 \mathrm{~B}$ & $20 \mathrm{C}$ \\
\hline $\mathbf{A}[\mathrm{AAAAA}] 2 / 173$ & B [BBBAA] 2/102 & C [CCCBB] 14/147 \\
\hline $\mathbf{A}[\mathrm{AAAAA}] 2 / 217$ & B [BBBAA] 4/95 & $\mathrm{C}$ [CССВB] 3/87 \\
\hline $\mathbf{A}$ [AAAAA] 3/182 & B [BBBAA] 5/129 & $\mathrm{C}$ [CССВB] 6/139 \\
\hline $\mathbf{A}$ [AAAAA] 3/217 & B [BBBBA] 8/260 & $\mathrm{C}$ [CCСBВ] 6/167 \\
\hline A [AAAAA] 5/195 & B [BBBBA] 10/189 & $\mathrm{C}$ [CССВB] 6/71 \\
\hline A [AAAAA] 5/242 & B [BBBBA] 2/125 & C [CСССВ] 8/127 \\
\hline A [AAAAA] 5/258 & $\mathbf{B}[\mathrm{BBBBA}] 2 / 72$ & C [CСССВ] 9/208 \\
\hline $\mathbf{A}$ [AAAAA] 7/250 & $\mathbf{B}[\mathrm{BBBBA}] 2 / 93$ & C [CCCCC] 10/114 \\
\hline $\mathbf{A}$ [AAAAA] $7 / 275$ & $\mathbf{B}[\mathrm{BBBBA}] 5 / 121$ & $\mathbf{C}[$ ССССC] 12/127 \\
\hline $\mathbf{A}$ [AAAAB] 10/146 & B [BBBBA] 5/144 & $\mathbf{C}[\mathrm{CCCCC}] 2 / 62$ \\
\hline $\mathbf{A}$ [AAAAB] 10/70 & B [BBBBA] 6/145 & $\mathbf{C}[\mathrm{CCCCC}] 3 / 73$ \\
\hline $\mathbf{A}[\mathrm{AAAAB}]$ 4/206 & B [BBBBA] 8/192 & C [CCCCC] 4/43 \\
\hline $\mathbf{A}$ [AAAAB] 3/152 & $\mathbf{B}[\mathrm{BBBBA}] 6 / 97$ & C [CCCCC $]$ 4/90 \\
\hline \multirow[t]{13}{*}{$\mathbf{A}[\mathrm{AAABB}]$ 4/127 } & B [BBBBB] 7/98 & C $[$ CCCCC $] 5 / 37$ \\
\hline & B [BBBBB] 3/91 & C [CCCCC $] 5 / 62$ \\
\hline & B $[\mathrm{BBBBB}] 4 / 128$ & $\mathbf{C}[\mathrm{CCCCC}] 5 / 70$ \\
\hline & B [BBBBB] 4/95 & C [CCCCC] 6/59 \\
\hline & B [BBBBB] 6/92 & $\mathrm{C}[\mathrm{CCCCC}] 6 / 63$ \\
\hline & B [BBBBB] 7/99 & $\mathrm{C}[\mathrm{CCCCC}]$ 7/111 \\
\hline & B [BBBBB] 7/149 & C [CCCСC] 9/109 \\
\hline & B [BBBBC] 10/186 & \\
\hline & B [BBBBC] 12/237 & \\
\hline & B [BBBBC] 6/133 & \\
\hline & B [BBBCC] 8/76 & \\
\hline & B [BBBCC] 9/69 & \\
\hline & B [BBBCC] 1/69 & \\
\hline
\end{tabular}

\subsection{Discussione}

Provando finalmente a discutere queste annotazioni, osserviamo ora (Figura 4) percentualmente la distribuzione delle diverse tipologie di testi nel sottocorpus. La maggioranza non assoluta dei testi è stata valutata basica; seguono i testi ascrivibili alla categoria di prebasico; il numero minore di testi, infine, è stato valutato postbasico. 
Figura 4. Percentuale di distruburione di testi giudicati basici e pre-e post-basici nel sottocorpus

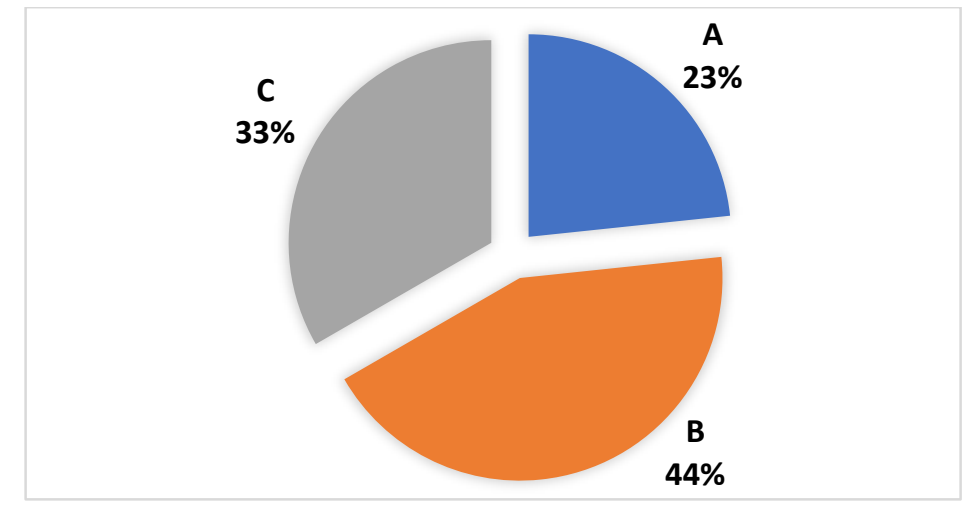

Riportiamo poi, infine, la media di ipotesi di intereferenza per ogni testo nelle diverse tipologie.

- Media transfer per ogni testo $C=6,5$

- Media transfer per ogni testo $B=5,7$

- Media transfer per ogni testo $A=5$

$\grave{E}$ un dato di grande rilievo che, come si vede, tra le diverse tipologie di testi (A, B, C) i valori non siano lontani, seppure prevedibilmente differenti.

Figura 5. Media di forme riconducibili a transfer per ogni testo suddivise per tipologia (basico, pre- e post-basico) di testi

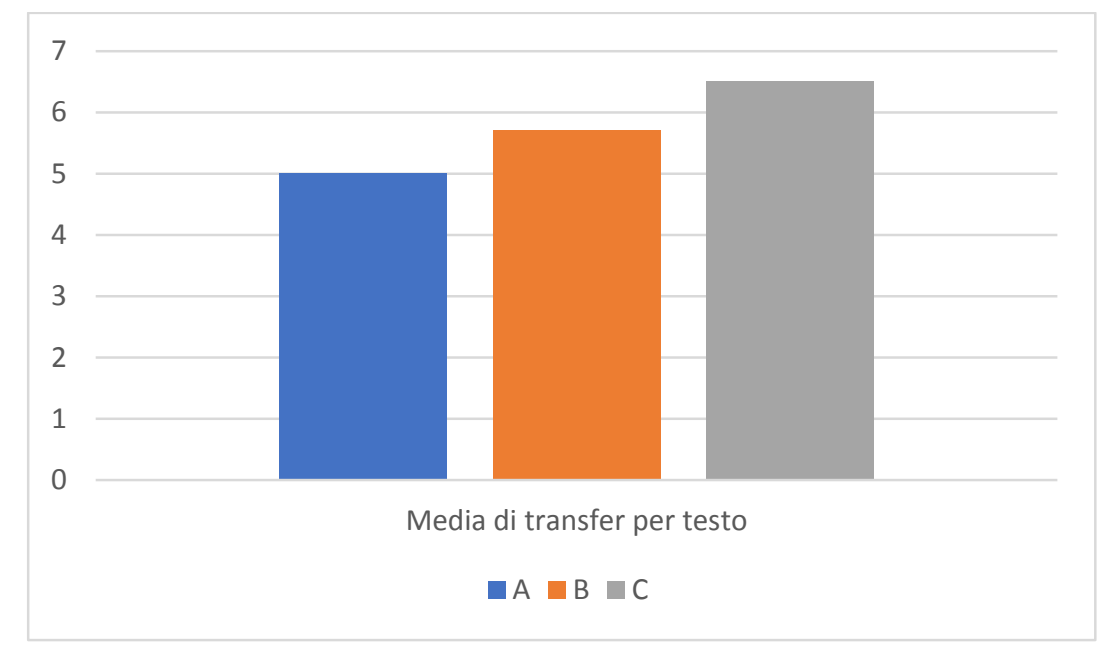

Ci pare soprattutto utile, ora, osservare la presenza massiva di fenomeni riconducibili a interferenza nei testi giudicati basici quanto in quelli prebasici e la presenza significativa di tali fenomi, seppure certamente in misura un po' inferiore, in quelli postbasici. In generale, $\mathrm{i}$ fenomeni che abbiamo preso in considerazione sono cioè osservabili nei testi giudicati $\mathrm{C}$, quanto nei $\mathrm{B}$, quanto negli $\mathrm{A}$.

Del resto, procedendo in modo puramente qualitativo e osservando a confronto i testi che abbiamo riportato ad esempio qui in alto (Figura 2), possiamo fare una serie di osservazioni linguistiche: 
(1) il testo giudicato basico B contiene molti elementi attesi di interferenza;

(2) in A e B i transfer non intaccano la comprensibilità del lessico;

(3) in C la storpiatura lessicale ("lita", "cali") si inserisce in un contesto di disordine testuale che coinvolge (come è caratteristico dei testi prebasici) anche le categorie grammaticali più o meno in relazione a fenomeni di trasfer stricto sensu (come il verbo avere in "due persona chi una problema").

Notiamo poi che nell'annotazione degli elementi di transfer non si è fatta alcuna distinzione tra transfer negativo oppure no. $\mathrm{Ci}$ sono degli elementi, infatti, come la probabile tense neutralization dell'esempio di testo basico riportato (B), in cui le forme annotate (sta, chiede) sono testualmente accettabili anche per l'italiano come presente narrativo.

Queste considerazioni possono essere estese a tutti i testi del sottocorpus. In alcuni casi, del resto, come [t39], sono stati valutati basici, e quindi comunicativamente efficaci, testi che sembrano dei veri cataloghi di interferenze da parte di L1 (a livello sintattico, morfologico, fonetico/ortografico).

Un'altra considerazione che ci pare importante è che nei testi giudicati $C$ sono sempre presenti elementi morfologici rapportabili a una dimensione di italiano postbasico (verbi coniugati, presenza di articoli e preposizioni etc.). Tale fatto ci deve fare riflettere, come si è già detto, non solo su quanto il paradigma della BV debba essere adattato secondo il modello proposto nel diagramma precedente (Figura 1) a un contesto di apprendimento guidato, ma anche sulla necessità, più generale, di misurare bene i risultati della linguistica acquisizionale in rapporto a tali contesti.

Ci sono pochi testi $[\mathrm{t} 21, \mathrm{t} 26, \mathrm{t} 60$ ] che costituiscono eccezione (certamente postbasici e vicini alla LT), ma nemmeno in questi casi mancano ipotesi di interferenza.

Proponiamo, dunque, a partire dall'osservazione di questi dati, una prima e del tutto provvisoria risposta alle domande di ricerca. Innanzitutto, si può dire che, apparentemente, nei testi presi in esame nel corpus VALICO, gli eventi di interferenza considerati (alcuni fenomeni tipici dell'interlingua di arabofoni apprendenti italiano L2) non sembrano correlati (cioè legati in modo biunivoco) alla funzionalità basica dell'interlingua. Questa caratteristica della lingua basica è del resto stata già implicitamente accolta dagli studiosi; ad esempio da Vedovelli (2002), che ha notato, descrivendo le varietà iniziali di italiano L2, come il livello basico fosse certamente contraddistinto anche dalla presenza di interferenze.

Sulla base di questa considerazione generale occorre però osservare che, anche quando vi è una relazione tra interferenza e giudizio di efficacia/basicità/sufficienza comunicativa, tale relazione è certamente posta al confine tra morfologia e lessico, quello cioè per cui la distorsione o l'adattamento producono una forma lessicale lontanissima dalla varietà target, e il cui significato è irrecuperabile anche a partire dal contesto. Si vedano ad esempio le forme incomprensibili "lacazzione" [t30], "delancarto" [t31], "nafikha" [t32]; e valga da controesempio una forma come "bagaglie" [t35]", la quale non intacca invece la comprensione. Tale relazione è ovviamente di natura prevalentemente quantitativa, cioè legata al numero di parole non comprensibili all'interno del testo e alla loro distribuzione, molto più che alla presenza di un singolo elemento. Si tratta dunque di una questione di competenza da riportare al sistema dell'apprendente e non alla singola forma. Il che rafforza la definizione sfocata delle categorie pre/post/basico che abbiamo esposto sopra (Figura 1).

${ }^{11}$ È certamente possibile che, in questo come in altri casi, la perturbazione morfologica rappresenti il risultato di un'interferenza prodotta anche da altre lingue, come il francese. 
È appena il caso di dire quanto occorra, tuttavia, certamente ampliare il campione di indagine, estendendo ad altri corpora il metodo, e ampliando anche il numero di giudizi di comprensibilità, per verificare questa ipotesi.

Rispetto alla seconda domanda di ricerca, la risposta - certamente del tutto parziale e nulla più che ipotetica - è che il paradigma della BV sia fortemente vincolato alla LT e meno alla L1. Senza ovviamente alcuna pretesa di affrontare in questa sede la diatriba generale tra i sostenitori e i detrattori di un approccio (classicamente) contrastivo, appare piuttosto evidente, a partire dalla lettura di questi primi dati, che - almeno per ciò che concerne la formazione di elementi tipicamente ascrivibili a una varietà di base - il meccanismo interferenziale, e quindi il peso della L1 (o di altre lingue precedentemente apprese), non abbia un ruolo definitorio e non influisca sulla basicità (e quindi sull'efficacia funzionale) o meno dellinterlingua. Fatto salvo ovviamente ciò che già molti anni fa rilevava Giacalone Ramat (2003: 348), sulla scorta di Klein e Perdue (1997) ${ }^{12}$, ovvero che i principi funzionali della $\mathrm{BV}$ «sembrano riflettere proprietà universali del linguaggio [...] e obbedire a principi organizzativi simili e indipendenti, almeno in parte, dalla lingua di partenza e da quella di arrivo», le forme della BV saranno piuttosto, nella dinamica di un processo acquisizionale evolutivo, il frutto di una tendenza alla semplificazione tipica delle varietà di apprendimento, rispetto alla quale i fenomeni di interferenza rappresentano solo uno dei molti fattori di influenza.

\section{OSSERVAZIONI CONCLUSIVE}

Due osservazioni conclusive che, ancora sulla base della lettura dei dati raccolti, si pongono soprattutto come domande per una ideale prosecuzione di questo studio. La prima considerazione è che bisogna forse ancora ripensare, in modo più sistematico, le categorie del paradigma della $\mathrm{BV}$ in relazione a contesti guidati di apprendimento linguistico (Figura 1). In scritti prodotti da apprendenti in contesto perlopiù guidato come quelli presi in esame in questo contributo - osserviamo, infatti, anche in testi certamente inefficaci dal punto di vista comunicativo, fenomeni morfologici congruenti con la VT; del tutto specularmente alla presenza di forme prebasiche in testi giudicabili postbasici.

In secondo luogo, è elemento di profondo interesse, forse non ancora sufficientemente esplicitato, il rapporto tra scrittura e varietà nel contesto dell'arabofonia: se è stato più volte osservato quanto, per il parlante arabofono, la scrittura sia tradizionalmente legata quasi esclusivamente alla varietà di prestigio, sarebbe tuttavia interessante riflettere sull'evoluzione della scrittura della varietà di apprendimento, anche in relazione al relativamente nuovo contesto della scrittura digitale legata, soprattutto, ai social network; un tema, questo, recentemente preso in considerazione da Levanoni (2018: 3):

The penetration of mass media, such as newspapers, radio and television, created a novel combination of literary Arabic and spoken Arabic. However, the real revolution in Arabic should be attributed to the expansion of the digital space, especially the use of online social networks where, for the first time in the history of spoken Arabic, the various dialects are being written rather than being limited to speech as they w ere in the past.

12 «Independent of the specifics of source and target language organisation» (Klein, Perdue, 1997: 304), come abbiamo già ricordato. 
Riprendendo alcune delle idee espresse nei primi paragrafi, e alla luce di queste domande, si potrebbe forse dare forza all'ipotesi che il modello interlinguistico scritto del parlante arabofono abbia una spiccata natura basica soprattutto nello scritto in L2 funzionale, ovvero un modello tutto orientato all'efficacia comunicativa (e al riparo dalla necessità di accuratezza conservativa dello standard). Questa idea - che nel quadro della nostra ricerca è, appunto, ancora solo un'ipotesi - potrebbe essere corroborata utilmente dai dati di altri studi (come, ad esempio, quello già citato di Bosco, 2004) da cui emerge che gli apprendenti arabofoni hanno sia una forte motivazione all'apprendimento dell'italiano scritto (e non solo e non tanto orale), sia una chiara motivazione funzionale: il lavoro e l'inserimento. In altre parole, forse si può parlare, oggi, di una nuova idea della scrittura, diversa da quella فصحى dell'arabo standard e più orientata da un criterio funzionale: quindi, nel caso della produzione in L2, con una natura fortemente basica. Entro un continum sociolinguistico in evoluzione e cambiamento seppure all'interno di un modello fortemente conservatore (Albirini, 2016: 326 e sgg.), si potrebbe provocatoriamente dire che nel mondo arabo (come in quasi qualsiasi altro contesto culturale) si scrive oggi forse di più e un po' peggio; cosicché anche la produzione scritta in BV sembra sempre più accettabile.

In conclusione, in un mondo in cui la scrittura ricopre molte delle funzioni tradizionalmente rivestite dalla comunicazione orale ${ }^{13}$, sarebbe importante chiedersi quanto peso assumano proprio le varietà nella produzione scritta e come cambi, di conseguenza, anche la natura della scrittura in L2.

\section{RIFERIMENTI BIBLIOGRAFICI}

Abi Aad A. (2006), "Insegnare l'italiano ad arabofoni, variazioni e interferenze", in Rassegna italiana di linguistica applicata, XXXVIII, 2-3, pp. 323-343.

Al-Ali A., Al-Oliemat A. (2008), "L'apprendimento dell'italiano come lingua straniera da parte degli apprendenti arabofoni in un contesto guidato: i tempi verbali italiani nel modo indicativo", in Studi di Glottodidattica, 3, pp. 1-15.

Albirini A. (2016), Modern Arabic Sociolinguistics Diglossia, variation, codeswitching, attitudes and identity, Routledge, New York.

Anghelescu N. (1993), Linguaggio e cultura nella civiltà araba, Zamorani, Torino.

Balboni E. P. (1999), Parole comuni culture diverse, Marsilio, Venezia.

Bernini G. (1988), "Questioni di fonologia nell'italiano lingua seconda", in Giacalone Ramat A. (a cura di), L'italiano tra le altre lingue. Strategie di acquisizione, il Mulino, Bologna, pp. 77-90.

\footnotetext{
${ }^{13}$ In proposito si è parlato recentemente di rivoluzione anche linguistica della cultura araba, proprio in merito all'ingresso della scrittura nella varietà/dialetti, ad esempio in conseguenza delle Primavere arabe e alla rivolta libanese (cfr. gli studi riportati dai ricercatori di OASIS:

https://www.oasiscenter.eu/it/libano-proteste-rivoluzione\#_ftnref6;

https://www.oasiscenter.eu/it/diglossia-nel-mondo-arabo;

https://www.annahar.com/english/article/1066287-the-revival-of-the-arabic-language-amid-the-

lebanese-revolution); c'è inoltre una certa diffusa attenzione al modo in cui il texting (la scrittura digitata, soprattutto nelle piattaforme di messaggistica) ponga gli arabofoni nella necessità e nella possibilità di scrivere nella varietà tradizionalmente orale (https://lingualism.com/arabic/arabs-say/do-arabs-write-intheir-dialects-of-arabic/) e al cosiddetto "Arabizi” (Yaghan, 2008).
} 
Bernini G. (2005), "La seconda volta. La (ri)costituzione di categorie linguistiche nell'acquisizione di L2", in Costamagna L., Giannini S. (a cura di), Acquisizione e mutamento di categorie linguistiche, Il Calamo, Roma, pp. 121-150.

Bettega S., Russo L. (2017) “Interferenza della L1 nell'apprendimento del sistema verbale italiano da parte di studenti arabofoni", in Corino E., Onesti C. (a cura di), Italiano d'apprendenti. Studi a partire dai corpora VALICO E VINCA, Guerra, Perugia, pp. 41 59.

Bosco O. (2004²), "Le motivazioni degli immigrati stranieri all'apprendimento della lingua italiana in contesto scolastico", in Vedovelli M., Massara S., Giacalone Ramat A. (a cura di), Lingue e culture in contatto. L'italiano come L2 per gli arabofoni, FrancoAngeli, Milano, pp. 141-152.

Boussetta A. (2006), Le influenze della lingua e della cultura materna sull'insegnamento dell'italiano agli studenti marocchini:

http://www.ilnostropianeta.it/wp-content/uploads/2010/09/italiano-perarabofoni-boussetta.pdf.

Branca P., Santerini M. (2008), Alunni arabofoni a scuola, Carocci, Roma.

Brincat G. (2021), "L'acquisizione imperfetta di una lingua adstrato. L'italiano degli analfabeti a Malta dal Settecento al Novecento", in Caruana S., Chircop K., Gauci P., Pace M. (a cura di), Politiche e pratiche per l'educarione linguistica, il multilinguismo e la comunicazione interculturale, Ca' Foscari, Venezia, pp. 15-27.

Brustad K. (2000), The Syntax of Spoken Arabic, Washington University Press, Washington.

Ceglia L. (2000), "Marcatezza e acquisizione di tratti. Il caso /p/ /b/ nell'italiano di arabofoni", in Cristofaro S., Putzu I. (a cura di), Languages in the Mediterranean area. Typology and Convergence, FrancoAngeli, Milano, pp. 43-63.

Colussi E., Cuciniello A., D’Annunzio B. (2014), Guida alla classe plurilingue. Insegnare italiano L2 ad adulti arabofoni e sinofoni, ISMU, Milano.

Comrie B. (1997), "On the origin of the Basic Variety", in Second Language Research, 13, 4, Special Issue on Introducing the Basic Variety, pp. 367-373.

Corino E., Marello C. (2017), Italiano di stranieri. I corpora VALICO e VINCA, Guerra, Perugia.

Corino E., Onesti C. (a c. di) (2017), Italiano di apprendenti. Studi a partire da VALICO $e$ VINCA, Guerra, Perugia.

Cuzzolin P. $\left(2004^{2}\right)$, "Percezione del contatto di lingue: arabo classico, arabo moderno, italiano, dialetto", in Vedovelli M., Massara S., Giacalone Ramat A. (a cura di), Lingue e culture in contatto. L'italiano come L2 per gli arabofoni, FrancoAngeli, Milano, pp. 89-110.

Deheuvels L.-W. (2010), Grammatica araba, edizione italiana a cura di Ghersetti A., Zanichelli, Bologna.

Della Puppa F. (2006), Lo studente di origine araba, Guerra, Perugia.

Della Puppa F. (2007), "Dall'analisi del contatto arabo-italiano L2 alle implicazioni glottodidattiche", in Studi di Glottodidattica, 3, pp. 22-40.

Della Puppa F. (2008), "Analisi del contatto arabo-italiano L2 su testi scritti in contesto di insegnamento formale", in ITALS, 16, pp. 7-27.

Della Puppa F. (2014), L'italiano non è difficile. Eserciz̨i di italiano per arabi, Loescher, Torino.

Della Puppa F. (2009), "Il ruolo della L1 nella formazione degli errori in L2: il caso del contatto arabo-italiano", in ITALS, 20, pp. 27-45.

Eddahani C., Dlimi S. (2019), Rappresentarioni linguistiche dellitaliano LS in Marocco, in Bollettino ITALS, febbraio 2019, pp. 1-23:

https://www.itals.it/sites/default/files/pdf-

bollettino/febbraio2019/Charifa_Eddahani_Samira_Dlimi.pdf. 
Elgheriwi A.E.A., (2017), L'apprendimento e l'insegnamento dell'italiano come LS per gli arabofoni. Un'indagine svolta in Libia, Tesi di dottorato, Ciclo XXIX, Università degli studi di Palermo.

Giacalone Ramat A. $\left(2003^{8}\right)$, "Italiano di stranieri”, in Sobrero A. (a cura di), Introdu₹ione allitaliano contemporaneo. La variazione e gli usi, Laterza, Roma-Bari, pp. 341-410.

Hetzron R. (ed.) (1997), The Semitic Languages, Routledge, London-New York.

Holes C. (2004), Modern Arabic: Structures, Functions and Varieties, Revised Edition, Georgetown University Press, Washington.

Jordens P. (1997), "Introducing basic variety", in Second Language Research, 13, 4, Special Issue on Introducing the Basic Variety, pp. 289-300.

Klein W., Perdue C. (1997), “The Basic Variety (or: Couldn't natural languages be much simpler?)", in Second Language Research, 13, 4, Special Issue on Introducing the Basic Variety, pp. 301-347.

Larcher P. (2008), "Al-lugha al-fuṣhâa: archéologie d'un concept «idéolinguistique»”, in Langues, religion et modernité dans l'espace musulman, 124, pp. 263-278.

Levanoni M. (2018), “An Arabic Language Revolution”, in BeeHive, 6, 8, pp. 3-6.

Martari Y. (2021), "Varietà di base e interlingue. Spunti di riflessione per l'insegnamento linguistico", in Gallina F. (a cura di), Itinerari di formazione: spunti di riflessione per $i$ docenti di lingue seconde, ETS, Pisa, pp. 45-70.

Martari Y. (in preparazione), "L'emergenza della varietà di base. Teoria della complessità e complessità dell'apprendimento linguistico".

Meisel M. J. (1997), "The L2 Basic Variety as an I-language”, in Second Language Research, 13, 4, Special Issue on Introducing the Basic Variety, pp. 374-385.

Ryding K. C. (2005), A Reference Grammar of Modern Standard Arabic, Cambridge Unversity Press, Cambridge.

Pallotti G. (2017), "Applying the interlanguage approach to language teaching", in International Review of Applied Linguistics in Language Teaching (IRAL), 55, 4, pp. 393412.

Salem A. (2017), Prima lingua e identità. Il caso della generazione degli arabofoni in Italia, Tesi di dottorato XXX ciclo, Tutor Francesco De Renzo, La sapienza, Roma.

Suleiman Y. (2003), The Arabic Language and National Identity. A Study in Ideology, University Press, Edinburgh.

Veccia Vaglieri L. ([1961] 2011), Grammatica teorico pratica della lingua araba, Istituto per l'Oriente, Roma.

Vedovelli M. (2002), "Italiano come L2", in Lavinio C., Sgroi C. S. (a cura di), La linguistica italiana alle soglie del 2000 (1987-1998 e oltre), Bulzoni, Roma, pp. 161-212.

Vedovelli M., Massara S., Giacalone Ramat A. (a cura di) (2004²), Lingue e culture in contatto. L'italiano come L2 per gli arabofoni, FrancoAngeli, Milano.

Versteegh K. (1997), The Arabic Language, University Press, Edinburgh.

Villarini A. (2021), Didattica delle lingue straniere, Bologna, il Mulino.

Yaghan M. A. (2008), “'Arabizi': A Contemporary Style of Arabic Slang”, in Massachusetts Institute of Technology Design Issues, 24, 2, pp. 39-52. 


\section{APPENDICE}

\section{Testi citati di arabofoni estratti dal Corpus VALICO e annotati}

(t21, t23, t25, t26, t28, t30, t31, t32, t35, t38, t39, t60)

I testi sono annotati manualmente con indicazione dei principali fenomeni ipoteticamente riconducibili a interferenza (in grassetto) su:

- Sistema vocalico e dittongazione

- Perturbazione consonantica (es. $v>b, g l>\emptyset$

- Geminate

- Posizione/uso articolo

- Coniugazione del sistema verbale complesso dell'italiano

- Sovraestensione participio ("participio attivo")

- Sovraestensione imperfetto

- Funzione deittica del verbo (difficoltà presente $>$ futuro $>$ passato)

- Ordine Determinato-Determinato

- Uso copula del verbo

- Tense neutralization

- Uso verbo avere

- Possessivo e "stato costrutto"

- Frase relativa

Non segnaliamo qui le interferenze dovute al sistema grafico (maiuscole/minuscole e spaziatura). Segnaliamo invece in grassetto corsivo alcuni prestiti e alcune significative ipotesi di interferenza da altre lingue (spagnolo e inglese) diverse dalla lingua madre e dalla lingua target.

Riportiamo in conclusione a ogni singolo testo la valutazione complessiva e analitica dei lettori (ex. A $[\mathrm{AAAAA}])$ e il numero di ipotesi di transfer individuato in rapporto al numero di parole (n/nnn).

$21 \mathrm{~L}$ ' altro giorno due uomini camminavano sul marciapiede, il primo veniva di sinistra e l' altro di destra . Tutti i due avevano molti bagagli . Il primo aveva una bicicletta sulla quale c' era di che cosa a mangiare ed a bere. Era un uomo basso con i capelli lunghi, portava un cappello, degli occhiali da sole, una camicia ed un pantalone corto . Con una mano teneva la bici e dell' altro un piccolo cane . L' altro uomo era alto con i capelli corti, portava una giacca ed un pantalone . Aveva nelle sue mani una borsa di supermercato ed un pal- lone di calcio. Lui teneva anche un grande cane. I due uomini intravano in una collisione. Tutti i due bagagli e tutto il cibo erano per terra . Cominciavano a parlare con alte voce . $C^{\prime}$ era una donna, chi guardava il primo uomo , gli ha preso i suoi palloni e se ne andava senza dire qualcosa . A [AAAAA] 2/173

23 L' altro giorno due uomini camminavano sul marciapiede. Il primo è era un uomo che ha avrà una trentanta, era un tipo sportivo. Lui aveva appena fatto la spesa, camminava con la sua bicichletta anche tutto le cose che avea comprato. Ha comprato esatamente del pane, dell' acqua e della verdura, stava aspettando una perfetta giornata è e per questo motiva ha port aveva portato il suo cane con lui . Il secondo era un tipo robusto, il suo stylo di vestirsi era un pò volgare . Camminava così con degli occhiagli da sole sulla fronte, portava anche una grande centura, su cui c' e l' immagi un' immagine. Il secondo aveva comprate molte cose anche e portava anche il suo cane che un pò cattivo respetto all' altro. I due uomini sembravano da due mondi diversi, e in un momento queste questi due mondi si incontravano sono incontrati . Quando loro si sono caduti nello stesso momento, tutte le cose che avevano hanno comprato erano per terra e non sapevano quelle quello per il primo e quelle per il secondo. Il secondo era 
molto arrabiato con l' altro e ha voluto restituire la sua spesa. Molte cose comprate erano scadute en e non servano ora per niente. Ciascuno di loro stava cercando a trovare le sue cose, ma in quella ambia caotica, nessuno provava a trovare quello che aveva comprato . A [AAAAA] 7/250

25 L' altro giorno due uomini camminavano sul marciapiede, L' uno di due aveva un cane e un bicicletta e l' altro aveva also un cane . il primo uomo e b meno alto quel di due . al contrario il secondo e molto alto e più forte . alle proviso gle gli due uomini confrontavano fortemente l' uno a l' altro e tutti li bagagi erano sulla terra $\mathbf{B}[\mathrm{BBBCC}] 1 / 69$

26 L' altro giorno due uomini camminavano sul marciapiede, il primo aveva una bicicletta e sembrava rientrare del mercato perchè la sua bici era caricata di bottiglie, legumi e pane ; di più, l' uomo aveva uno zainetto dove erano attacati due palloni e teneva con se un cane piccolo magro come il suo padrone. Invece il secondo uomo era più grande con una aria minaciante, anche lui sembrava ritornare del super mercato si vedeva dai sacchi che aveva nelle mani, anzi c' era anche un pallone e una un grande zaino e una piccola borsetta, anche lui aveva con se un cane ma quest' ultimo era grande forte e come il suo padrone aveva l' aria minaciante e sembrava vi molto forte visto che il suo padrone lo teneva con una catena . all incrocio dei due marcia piedi i due uomini si sono scontrati e tutto quello che era con loro nelle borse e nei zaini si e disperso per terra e nell' aria , si poteva vedere i salumi gli occhiali il pane dei * libri volare in aria . con que tutto questo casino i due uomini cominciavano a litigare e a battersi con tutto quello che poteva caderli su su le mani, libri salumi ed altro . A [AAAAA] 3/217

28 L' altro giorno due uomini camminavano sul marciapiede erano tutti i due carichi di spese che avevano comprato un giovane ragazzo con la sua bicicletta carica di spese che aveva comprato al supermercato ( latte, olio, frutta e anche una salsicia ). Era in compagnia con suo piccolo cane - Rientrava tranquillamente a casa cuando all' improviso ha sbattuto contro un' altro uomo che veniva dalla parte opposta del marciapiede. Era anche lui stracarico di roba diversa sulla sulle spalle, nelli mani, con un cane ( anche lui). Una signora affaciata alla finestra davante la scena di sbattimento dei due uomini ne aprofitta per racogliere i due ( palloni) palloni . (mentre i due uomini ( non ). Invece di racogliere la loro spesa spersa ? da per tutto, loro si misero a litigare con le salsice. A [AAAAB] 10/146

30 L' altro giorno due uomini camminavano sul marciapiede l' ultimo vendettore si chiama Carlo, l' altro si chiama Paolo. Carlo è molto gentile si veste beno bene accompagnato con un canino, guidare una bicicletta piena di cose exxp bottiglie . late ... L' altro sembra un pò strano con i suoi vestiti anche il suo cane è bruto porta adosso tante cose strane come lui . Le due sono incontrati , Paolo è molto nervoso è batti Carlo c'è sono uno un litigio allora gli due cane sfruttare lacazzione è mangiare tutti li cose . B [BBBBB] 4/95

31 Uno uomo gigante chi sembra Come uno delancarto, con i suoi affari e il suo cane ha in contrato in giro uno uomo magro chi a i suoi affari e spese con il suo piccolo cane, lincontra è trasformata in una battaglia tra le due uomi, le loro cose e affare sono and in disordine sul la strada, subito una donna che è stata a casa sua guardava il conflitto della finestra della sua casa, non è contenta di quale che ha visto perchè non ci un équilibro di due corpi dei uomi che ci sono litigati . B [BBBAA] 2/102

32 ci sono due uomini caminano sulla strada uno di loro camino a sinistra l' altro a destra con i piedi e porta con sè una Botiglia di late un pò grande un cane e sul zham spalle quel un zaino e laltro a destra porta con sé una bicicleta e sul spalle anche un ziano ma nella sulla bicicleta porta un sacheto in cui dei pani e nel suo mano un cane. nel momento l' uomo che porta una bicecleta é sei tornato a casa c'è una donna guarda da una finestra. Questi due uomini caminano così ed improvisamente si incontrano un di loro sta per cadere p tutti le cose che loro portano quasi si espandano sulla terra e qualche cose saltare in aria . l' uomo che sta a sinistra lo guarda molto arabiato . nel quel volare nel momento in cui gli uomini combateno le due cani colgono 
lacazione ( mangiano il pane bevano il latte e la dona che guarda la scena dalla finestra porta il palloncino nafikha la bicileta è rotta . B [BBBBA] 8/192

35 L' altro giorno due uomini camminavano sul marciapiede uno con la sua biccicletta è si chiama " Gino " un venditore che sta facendo un giro per vendere le suoi merce in strada . nivece $l^{\prime}$ altro uomo sembra un vagabondo con il suo cane . e porta in mano molti bagaglie . e improvisamente le due uomini sono stati incontrati bruscamente . sono iniziati a combattere Mentre il vagabondo sta gridendo fortamente l' altro che sembra gentile e stato attaccato da lui per non batterlo anche i due cane stanno disputando . anche sono arrabiati . Ma la grande uomo e molto di più nervoso che il venditore. non ha smesso di battere e gridare ma c'è da l' altra parte una donna sta guardando della sua finestra e chi ha sfruttato della situazione perché ha preso un pallone forze per il figlio . B [BBBBA] 6/145

38 la prima figura e la seconda figura parlano di due personone il primo magro e l' altro grosso le due sono camminavano sul marciapiede . doncui il primo persone chiamo paolo qui returnerè di passageata qui avevo fare la spesa al mircato con pagari di prodotti come latto, mocaroni ...... le due persone urtare con la parlono a urtato e tigrano e tutte le li prodotti caderna par terra $\mathbf{C}$ [CCCCC] $5 / 70$

39 Una bella giornata di sole, Marco, ha pensato di andare al supermercato per fare la spesa . Al fine di fare la spesa, hai retornato a suo casa . E sul marciapide, c'è un altro ummo uomo che è stato molto strano e magro con il suo grande cane. Il grande uomo ha portato un sacco di cosa : un zaino pieni dei cosi, un succo, ballone. Unaltro Marco ha andato piano piano con il suo bicicletta e il suo piccolo piccolino cane. Alimpovise, le due ummeni uomeni si hanno scontrato , e così tante le cose hanno stato sul terra . le lo strano ummo uomo è stato molto arrabiato e ha fatto una litta con Marco, ma anchi il grande cane ha stato arrabbiata. Marco é stato molto paura, perche non ha volute fare bagari con gli altri e certamento con un umo più grande $\mathrm{e}$ miscolose di lui . Lei ha demmendato domendato l' un scasa di strano uomo. Ma lui non ha voluto accettare la scusa. Anchi il piccolino cane è stato paura, non ha voluto guardare al grande cane! Un gatto sul finestra ha stato anchi paura e è scapato . Alla fine Marco ha deciso di pagare una somma di soldi per l' uomo e andare a casa . B [BBBBC] 12/237

56 Siamo in una stazione di tram dove ci sono tante persone che stanno aspettando sia i loro amici , sia l' orario del viaggio . Dal Da un' un latto, ci sono due innamorati che uno di loro sta per viaggiare. Dall' altra parte, c'è una donna mentre sta prendendo il pranzo, passa un cane e fa cadere la tavola dove ci sono dei cibi. Quest' azione ha preso l' attenzione di tutti le persone che sono alla stazione. Dietro a questa donna, c'è della gente che sta salutando le loro membre, amici. In breve in questa stazione tutto sembra strano, ognuno fa una cosa, ma il cane ha attirato l' attenzione di tutto . B [BBBBA] 2/125

60 Una volta, c'è un uomo che ha una bicycletta e che sta camminando alla strada insieme al suo cane . Sulla sua bicycletta ci sono tante cose : delle bottiglie, palloni ecc . A fronte di lui , c'è una donna che sta osservando dalla sua finestra. Dall' altra parte, c'è un' altro uomone omone che sta camminando insieme al suo cane che sembra più grande dell' altro cane . l' omone indossa un pantalone con una giacca e una grande collana . le sue mani sono pieni di cose . a sinistra c'è un pallone e a destra c'è un sacchetto, Invece all' altro uomo, che è un' uomo magro porta un' occhiale nero con un sacchettino che è legato alla bicycletta. Improvvisamente, gli due uomini hanno fatto uno scontro. Tutto le cose stanno volando : i panni, le bottiglie, le mele che sono a terra, i due cani, uno su l' altro. Infine, è caduto successo un litigo fra questi uomini. Mentre gli due uomini stanno litigando, la donna che sta osservandola scena, ha preso il pallone e il gatto dall' altra parte ha preso i calzoni, tutti i due hanno approfitato di questo litigio litigo . A [AAAAA] 2/217 